\title{
Balancing energy strategies in electricity portfolio management
}

\author{
Christoph Möller \\ University of Karlsruhe and KIT, Schlossbezirk 12, D-76131, Karlsruhe, Germany \\ E-mail: christoph.moeller@statistik.uni-karlsruhe.de

\section{Svetlozar T. Rachev} \\ University of Karlsruhe and KIT, Schlossbezirk 12, D-76131, Karlsruhe, Germany \\ E-mail: rachev@statistik.uni-karlsruhe.de
}

\section{Frank J. Fabozzi}

\section{Contact author}

Yale School of Management, 135 Prospect Street, New Haven, CT 06511, USA

Telephone number: 001-(215) 598-8924

Fax number: 001-(215) 598-8932

E-mail: frank.fabozzi@yale.edu

\footnotetext{
Acknowledgment: Rachev gratefully acknowledges research support by grants from the Division of Mathematical, Life and Physical Sciences, College of Letters and Science, University of California, Santa Barbara, the Deutschen Forschungsgemeinschaft and the Deutscher Akademischer Austausch Dienst.

We further thank Dr. Dominik Möst from the Institute for Industrial Production at the University of Karlsruhe for valuable discussions and providing additional data.
} 


\begin{abstract}
Traditional management of electricity portfolios is focused on the day-ahead market and futures of longer maturity. Market participants use these markets to trade any excess production or consumption in their portfolio and balance their total position. Within limits, market participants can also resort to the balancing energy market to close their positions. The energy transactions on the balancing energy market can be separated into fluctuations caused by unpredictable events and strategic positions. In this paper, we determine strategic positions and identify corresponding economic incentives in an analysis of the German balancing energy demand. The strategies discussed contribute to an effective functioning of the electricity market.
\end{abstract}

Key words: electricity market design, balancing energy, strategic behavior, interchangeable marketplaces, electricity portfolio management

JEL: Q41, Q47 


\section{Introduction}

In the late 20th century, electricity markets were liberalized across the world. Since this restructuring, integrated companies have separated into specialized individual market participants and additional players have entered the market. All these players face not only the risk of a highly volatile energy market, but also the inner-market risk of the electricity market. Naturally, market participants want to actively trade and hedge this risk.

Alongside with traditional bilateral contracts, electricity exchange-traded contracts have emerged as instruments for these inner-market trades. Among these contracts the day-ahead futures contract is the principal instrument. This futures contract offers the shortest delivery period - often one hour - and serves as the reference price for longer-dated futures contracts. For this reason, the day-ahead futures contract is sometimes referred to as the spot contract. The properties of the day-ahead market and the market for futures written on day-ahead contracts are analyzed using various modeling approaches see, for example, (Geman and Roncoroni, 2006; Trück et al., 2007; Römisch and Wegner-Specht, 2005). In addition, the interdependence of the day-ahead market and the futures market is investigated see, for example, (Bessembinder and Lemmon, 2002; de Jong and Huisman, 2002).

The market for electricity consumption and delivery requires a marketplace for ancillary services so that blackouts may be avoided. The capacity reserve market is one such marketplace. Capacity reserve is provided by installations such as power stations or factories. The capacity of these installations can be traded on the electricity exchange as well as on the capacity reserve market. Thus, the day-ahead market and the capacity reserve market are interchangeable marketplaces for trading this capacity. It is recognized that the competition between these two marketplaces should be taken into account in formulating optimal bidding strategies in both trading forums see (Weigt and Riedel, 2007; Simoglou and Bakirtzis, 2008). In this paper, we extend this notion of interchangeable marketplaces to another ancillary services market, the balancing energy market.

The market design of the balancing energy market is a crucial component of electricity markets as it mediates between the liberalized futures- and day-ahead markets, and the natural monopoly of the grid and its operation see (ETSO, 2007). Nonetheless, the interplay of the balancing energy market with other marketplaces and the economic incentives that govern it have received little attention. This interplay is controversial. It has been argued that any use of the balancing energy market, apart from the settlement of imbalances caused by unpredictable events, might endanger system operation by adding the uncertainty of market participants' strategic positions. As a consequence the balancing energy market is reduced to a marketplace with a single focus on secure system operation and blackout prevention see (Belmans et al., 2009; ERGEG, 2006).

In the context of interchangeable marketplaces, we focus on two aspects of the balancing energy market. We first look at the balancing energy market as an alternative marketplace for reserve capacity. It offers market access to a wider 
and technically less demanding range of installations such as power stations or factories. Second, we investigate the balancing energy market as an alternative marketplace for electricity consumption and delivery. Because the balancing energy market is less responsive to short-lived events, it has the potential to dampen the effects of electricity price spikes on the electricity portfolio of market participants.

In view of the stated objective of increasing renewable generation in the future see (EU, 2008; U.S.Congress, 2009), these two aspects of the balancing energy market are becoming increasingly important. Often, renewables have a weather-dependent availability resulting in supply shocks. An efficient balancing energy market helps to tap the full reserve capacity potential in the market to cope with these shocks. Moreover, it mitigates the impact of these shocks on electricity prices.

In this paper, we provide evidence of the balancing energy market being utilized as an alternative market for both the electricity exchange and reserve capacity in Germany. The German market is chosen for this analysis due to the combination of its market design and generation stock. With its wind power market share of $15 \%$ and a clear incentive for active positions in the balancing energy market, Germany provides a suitable setting to observe the two aspects of the market described earlier.

The paper is organized as follows. Section 2 provides a brief review of balancing energy settlement schemes, followed by a description of the German market design and the motivation for the chosen setting in Section 3. Section 4 describes the data and introduces the proposed modeling approach. A quarterhourly pattern is analyzed in Section 5. Section 6 focuses on an hourly pattern, and the interdependence with the day-ahead market. At the same time, we look at the incentive structure leading to the observed patterns at these two timeframes. Additionally, we look at incentives for positions in the balancing energy market persistent over longer periods of time in Section 7. Section 8 summarizes our results and their implications.

\section{Balancing energy review}

Electricity is a practically non-storable commodity. Therefore, supply and demand have to be in exact equilibrium at all times. As a last resort a blackout (i.e., a static zero equilibrium) will follow should a dynamic equilibrium not be maintained within set boundaries. The equilibrium is monitored and maintained by the transmission system operator (TSO) in a certain control area. Everyone connected to the grid within this control area is required to provide the TSO with a forecast of feed-ins and withdrawals. Several market participants may pool this responsibility and form a balancing responsible party (BRP). BRPs are urged to trade any excess feed-in or withdrawals and provide a balanced forecast, so the task of maintaining the equilibrium reduces to balancing any unforeseen changes to the sum over all scheduled forecasts of all BRPs. How- 
ever, a BRP can always provide a balanced forecast by concealing excess feed-ins or withdrawals. This is an important point we will take up later.

In a liberalized market setting, the TSO will resort to up- and down-regulation bids to maintain balance. Activating an up-regulation bid will feed-in additional electricity into the network, while activating a down-regulation bid will withdraw electrical energy from the network. These bids are contracted by the TSO prior to the real-time balancing on the capacity reserve market, and can be provided by an altered operating state of both electricity producing and consuming installations. The TSO imposes pre-qualification standards of reliability and response time for these bids, so activated bids are reliable. These technical standards restrict market access to the capacity reserve market. Furthermore, reserve capacity is subdivided into three categories along a grading of these standards. Primary reserve provides an immediate and automated response to disturbances and therefore has to have the shortest response time. Secondary reserve and tertiary reserve serve as a back-up reserve to release the limited and expensive primary reserve after disturbance lasting for some time. In this way an adequate primary reserve capacity is secured at all times. This sketch of the balancing mechanism suffices in the context of this paper. For a more detailed description and alternative arrangements see (EU, 2005).

The service of balancing incurs costs which have to be borne by market participants. Some of these costs - mainly the cost of primary reserve - are socialized in grid tariffs.

The cost of lasting disturbances - primarily the cost of secondary and tertiary reserves - should be attributed to the originator, however. That is, a BRP consuming electricity in excess of its forecast should pay for this energy, while a BRP providing the network with electricity relative to its forecast should be compensated. Therefore, every BRP's deviation from its scheduled forecast is metered over certain settlement periods, and will be settled with the TSO. This energy is called balancing energy. In this paper, the deviation is defined as the difference between the actual load and the forecasted load. Accordingly, we will use the following sign convention in this paper: a positive sign means an undersupply and a negative sign means an oversupply. It is important to note that an individual BRP might deviate with opposite sign to the control area's net deviation (i.e., aid network stability with its deviation). However, this aid of network stability can be viewed as an incidental. From this point of view, it is dubious to have a BRP benefit from causing a disturbance that might as well have had an adverse effect on network stability.

Corresponding to this point of view and the possibly stabilizing effect of deviations, two general settlement schemes for balancing energy have emerged. These schemes are the single-price system that emphasizes the possibly stabilizing effect of a BRP's disturbance, and the dual-price system that emphasizes the general danger of disturbances for network stability. In a single-price scheme the TSO will set one price for both positive and negative deviations in each settlement period. Every BRP with a positive deviation during this period will pay this price, while every BRP with a negative deviation will receive this price for its energy deviation. Naturally, the balancing energy prices are high during 
periods with a positive net deviation as a majority of BRPs has to buy electricity on the balancing energy market. Equivalently, prices are low during periods with a negative net deviation. Thus, the single-price approach sets an incentive to deviate in the opposite direction to the net deviation in the control area (i.e., receive payments during high price periods and make payments during periods of low prices). In this sense, the balancing energy market is an alternative marketplace to the capacity reserve market. Just like in the capacity reserve market, a BRP with the flexibility to change the planned operating status of its installations for some period of time can make a profit. As stated above, a BRP also has the option to provide an only partially balanced forecast. This means speculating on the balancing energy price as the BRP's open position will be metered and then settled with the TSO. To evaluate this option, the day-ahead market price may serve as a reference price. If the spread between day-ahead and balancing energy price is positive, it is beneficial for BRPs to be in undersupply on the day-ahead market with a countering position in the balancing energy market, and vice versa for a negative spread. In this sense, the balancing energy market is an alternative marketplace to the day-ahead market. Note that the balancing energy price is set by the TSO after the settlement period. Consequently, the balancing energy market can only offer statistical-arbitrage opportunities.

The dual-price system is designed to suppress such statistical-arbitrage activity. In the dual-price settlement scheme, the TSO will set two prices for each settlement period, one price for positive deviation and one price for negative deviation. These prices can be understood as the single-price settlement scheme with an additional general fine imposed for deviation. The motivation for such fines is the additional variance that statistical-arbitrageurs might add to the balancing energy demand. From a TSO's perspective, any uncertainty about the balancing energy demand complicates its task of balancing and thereby potentially endangers system operation, as balancing energy is interchangeable with reserve capacity and day-ahead futures trades, it is hard to keep market participants from exploiting this. We illustrate this point by the example of two countries under a dual-price balancing energy scheme.

Kirschen and Garcia (2004) state that balancing energy is too expensive in the market in England and Wales. As a result, market participants keep their own reserve capacity rather than resorting to system reserves. Consequently, deviations will be actively managed even if an offsetting deviation exists somewhere else in the control area. This results in an inefficiently high allocation of reserve capacity (i.e., the market is in oversupply of production capacity). In contrast, Mielczarski et al. (2005) have argued that in Poland balancing energy is too inexpensive. So market participants use the system reserve to supply about $4 \%$ of total electricity demand (i.e., the market is in undersupply). These two examples demonstrate that the dual-price scheme does not guarantee the absence of arbitrage activity between the balancing energy market and dayahead or capacity reserve market.

In the following, we focus on a country with a single-price balancing energy settlement scheme. In general, the results and techniques should apply to a 
dual-price scheme as well. However, in this case the fine imposed for deviation has to be taken into account, for example as a transaction cost.

\section{German market design}

The details of electricity market designs are diverse, and necessarily reflect local specifics as generation stock and customary operation policies. Due to the limited interconnecting transmission capacity between control areas, this diversity will prevail in the future see (ETSO, 2007). In this section, we present details of the German electricity market that are relevant to the focus of this paper.

The central electricity marketplace in the German market is the European Energy Exchange (EEX). Most importantly, day-ahead contracts for all 24 hour intervals of the following day and corresponding base- and peak-load futures are traded on the EEX. These contracts are used as the underlying in longer-dated futures at the EEX, and often serve as a reference price in OTC trades. The entire German market is combined into one delivery location in these contracts.

In contrast, the German balancing energy market is subdivided into four control areas. The transmission system in these control areas is owned and operated by one of the four major players in the electricity market: E.ON AG (e.on), Rheinisch-Westfälisches Elektrizitätswerk AG (RWE), EnBW Energie Baden-Württemberg (EnBW) and Vattenfall AB (Vattenfall). All four control areas share a single-price balancing energy settlement scheme with quarterhourly settlement periods. So in Germany there are four individual control area net deviations and corresponding balancing energy prices for each of the 96 quarter-hour intervals of the day. However, there is a common property of these balancing energy prices. The spread between balancing prices during periods with positive and negative net deviation is four to five times the price of electricity in the day-ahead market. This spread results in a strong economic incentive for a BRP to deviate opposite in sign to the net deviation of its control area.

The design of the reserve capacity market has elements of both a single German marketplace and four individual marketplaces for the different control areas. Since 2007 the four TSOs have been allocating their primary-, secondary, and tertiary-reserve in a common auction. However, the location of facilities offered is considered in this auction to meet additional technical boundary conditions. More importantly in this context, secondary-reserve capacity is allocated in monthly auctions, and tertiary-reserve capacity is allocated in day-ahead auctions for six four-hour periods. Also secondary- and tertiary-reserve capacity prices are fundamental in the formation of balancing energy prices. Consequently, balancing energy prices will be less responsive to short-term supply shocks than the hourly day-ahead futures (i.e., balancing energy involves products with monthly and four-hourly averaging, while the day-ahead market is adapted to hourly averaging). In fact during periods of price spikes in the dayahead market it might be beneficial for a BRP to have a positive deviation, regardless of the control area's net deviation. 
The market design just described encourages two strategies involving active positions in the balancing energy market. First, a BRP should control its deviation so as to have the smallest correlation to the net deviation of its control area possible. Second, a BRP might try to exploit less responsive balancing energy prices in times of supply shocks. We will analyze such strategic positions in the balancing energy market in Sections 5, 6 and 7 .

It is important to note that such strategic positions are limited by gridaccess contracts. In these contracts BRPs are required to provide a balanced forecast and an abuse of balancing energy is prohibited. However, there is no definition of what a balanced forecast and abuse are. As an example, according to a sample contract of the German state agency, the mean deviation should not be "excessively" positive or negative. Also deviations must not show "conspicuously" arbitrage-like correlation with day-ahead exchange prices. On the other hand, sanctions to a detected violation of these rules are clearly defined in this sample contract. A BRP will be denied compensation during periods of a negative deviation, and charged double the day-ahead price in addition to the balancing settlement price during periods of positive deviation see (Bundesnetzagentur, 2006). So one can conclude that strategic positions in the balancing energy market are encouraged up to a level where they become conspicuous and excessive (i.e., a BRP has some flexibility in providing a balanced forecast).

We conclude this section by highlighting a few aspects of the German market design that distinguish Germany in the context of our analysis. Together with Scandinavia, Spain, Greece, and Hungary, Germany is among the minority of European countries featuring a single-price balancing energy settlement scheme. In the context of our analysis, a single-price settlement scheme is preferable as the complication attributable to a deviation fine can be ignored. In addition, the spread between balancing energy prices during periods of positive and negative net deviation is large, and thereby increases the economic incentive for strategic positions in the balancing energy market as noted earlier. This spread is smaller by one order of magnitude in the Norwegian market, for example, where an abundance of hydropower dominates the electricity market. Hydropower is easily controlled, which translates into cheap capacity reserve in this market. So the German market gives a more realistic picture of a common thermal-based electricity generation stock.

Additionally, the different fundamental periods of quarter-hour in the balancing energy market and one hour in the day-ahead market are advantageous to our analysis. As outlined in Section 2, the balancing energy market is an alternative marketplace to the day-ahead market and the capacity reserve market. Because the basic period is one hour in the day-ahead market, the balancing market interacts only with the capacity reserve market on the quarter-hourly timeframe. So the different fundamental periods allow analyzing the interaction with the two alternative marketplaces separately. 


\section{Model and data}

In this section, we present a model to forecast balancing energy demand in Germany. We identify four factors that influence balancing energy demand $\left(D_{B}\right)$ on different time scales. These factors are the gradient of load $(\nabla L)$, a dayahead market arbitrage incentive $(I)$, a technical incentive $\left(I_{\text {tec }}\right)$, and a varying general market position $(f)$. In addition, the model includes a non-predictable event risk $(\sigma)$. Assuming independence of the four factors, these factors can be modeled separately, yielding equation (1) as a model for balancing energy demand:

$$
D_{B}(t)=q(\nabla L(t))+h\left(I(t), I_{t e c}(t)\right)+f(t)+\sigma(t)
$$

There is a twofold separation in this model. First, the model separates strategic positions according to the time scale to which they are applied. These time scales are the quarter-hour interval, the hour interval, and positions taken over extended periods of time. Second, the model separates positions corresponding to the two alternative marketplaces, the day-ahead market and the capacity reserve market. All the model components - except for the $\sigma$ termdemonstrate that market participants are not using their best minimal variance forecast because predictable components remain.

It is important to note that the gradient of load and the day-ahead market arbitrage incentive are dependent on the load. In the latter case, this dependence is imparted by the price-load-dependence of electricity markets. Because of this dependence, it is possible to recover the general shape of the German load profile from the balancing energy data. Such a recovery of the load profile is an illustrative demonstration of the model's fitness and the underlying dependence. Because we use this technique in Sections 5 and 6 , we include a few facts about the German load profile. Figure 1 shows the load pattern of a typical summer and winter week obtained from 2006 data. As can be seen, there is a change from a higher general level in the winter to a lower level in the summer. Also, the shape of the first five cycles representing the weekdays differs considerably from the last two, the Saturday and the Sunday cycle. Within a day, the drastic increase from low night-time to high day-time levels in the morning, and vice versa in the evening, dominates the shape. One can also differentiate summer and winter load shapes by an additional load peak present in winter evening hours.

We conclude this section by describing our database. The analysis is based on the balancing energy demand data published by the four German TSOs. Publication of these data started on February 1, 2001 for RWETransportnetz Strom, December 1, 2001 for e.on Netz, January 1, 2002 for EnBW Transportnetze, and September 1, 2002 for Vattenfall Europe Transmission. For the purposes of this study, we combined the net deviations of all four German control areas to one hypothetical German control area, covering the years 2003 to 2008. This increases comparability to the single German day-ahead market and circumvents the issue of congestions between the control areas. Three additional data sources are used. First, we use the hourly electricity prices at the EEX 
Figure 1: Weekly load pattern (Monday to Sunday) in summer and winter

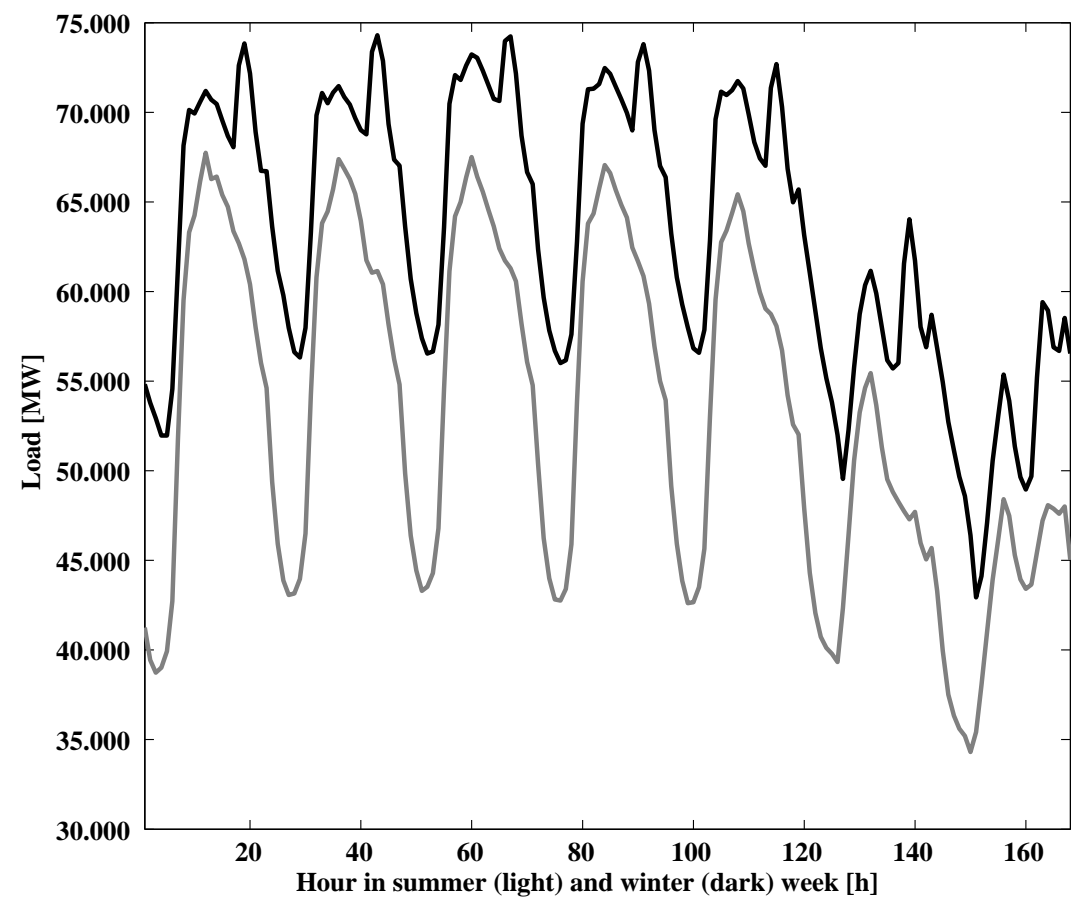


as a price reference. Second, the yearly consumption package provided by the Union for the Co-ordination of Transmission of Electricity (UCTE) serves as a proxy of electricity demand. Third, we resort to a 2004 load measurement in quarter-hourly resolution, provided by University of Karlsuhe IIP, for a detailed analysis on the quarter-hour timeframe.

\section{Quarter-hourly pattern}

Balancing energy is set with quarter-hour settlement periods in Germany, whereas the smallest contractual period on the day-ahead market is one hour. This discrepancy gives rise to a distinct pattern, which we appropriately term the quarter-hourly pattern. Let's consider the situation during an hour with an increasing load. The minimal variance forecast for this hour that is tradable in the day-ahead market is the mean load during that hour. With this forecast, the deviation will be negative in the first and second quarter of that hour and positive during the third and fourth quarter of that hour. Obviously, the same argument with opposite signs holds for a load decline. This effect will be more pronounced the higher the gradient of the load during that hour is, an effect that is also observed by Nailis and Ritzau (2006). Consequently, the effect can be modeled by the average load during the four quarter-hour periods $\left(\bar{L}_{q}(t)\right)$ and the average load during the corresponding hour $\left(\bar{L}_{h}(t)\right)$. We introduce the model in equation (2):

$$
q(\nabla L(t))=q \cdot\left(\bar{L}_{q}(t)-\bar{L}_{h}(t)\right) q \in[0,1)
$$

Here the parameter $q$ represents the electricity producers' ability to keep to their step function profile of hourly scheduled production. Should consumption and production change at the same rate, the factor will be zero. A value of one indicates a perfect step function of output, corresponding to an infinite ramping speed of power stations.

To test this model, we compare it to the empirical average pattern retrieved from the balancing energy data. We define this average pattern by the meanbalancing energy demand relative to the corresponding hour's mean value, conditional on the quarter-hour interval of a day. Figure 2 shows the resulting pattern using 2004 data. Here the four quarter-hour intervals of each hour are joined by lines to sort the 96 values. Note that by definition this pattern cannot be influenced by effects on hourly or even longer time scale, as each hour segment is centered on zero.

An estimation of the parameter $q$ using a 2004 load measurement yields $q=0.424$. Comparing the pattern retrieved from this model to the empirical pattern results in an $R^{2}$ equal to 0.8696 . This demonstrates the high explanatory power of the model for the quarter-hourly pattern. Additionally, fully exploiting the model's prediction reduced the sample variance by $12.03 \%$. To improve the illustration, the segments in Figure 2 are joined in Figure 3. The missing information on the gradient between two consecutive hours is estimated as the average of the adjacent segments. Figure 3 shows the joint pattern based 
on the load data scaled by our parameter estimate. Clearly the empirical pattern resembles the average German load profile. This is yet another indication of the model's fitness.

To further investigate the quarter-hourly pattern, we retrieve the patterns of all years in the interval 2003 to 2008. These patterns are used to predict the other patterns in this group in an out-of-sample analysis as shown in Table 1. The high $R^{2}$ values indicate the consistency of the quarter-hourly pattern. Moreover, the explanatory power tends to be higher for subsequent years. When using the pattern of the total sample and an additional scaling factor to predict the yearly patterns, we observe a diminishing amplitude of the quarter-hourly pattern (see Table 1). This finding is supported by Figure 4 which displays the joined yearly patterns for illustration.

It is important to note that the quarter-hourly pattern is driven by the consumption side of the market rather than the production side. By and large, the production side follows the step function dictated by hourly contracts, while consumption changes gradually. This brings about the quarter-hourly pattern. As there is no liquid market to trade electricity with sub-hourly delivery periods, there is practically no way to avoid the quarter-hourly pattern. For this reason, BRPs that are positively correlated to the load pattern (consumers) will incur additional cost, while BRPs that are negatively correlated to the load pattern (producers) will have a financial gain. So there is an economic incentive for BRPs to redistribute part of its load within an hour and obtain a negative correlation to the quarter-hourly pattern for that part of its load. Such a strategy is equivalent to buying electricity during periods with an expected lower net deviation and price, and selling it at higher prices during periods with an expected higher net deviation. This described strategy results in an intervention similar to that of reserve capacity and aids network stability. So on a quarter-hourly timeframe, the balancing energy market is an alternative marketplace to the capacity reserve market. However, in contrast to the capacity reserve market, there are no pre-qualification standards. Also there is no fixed compensation, but rather a statistical arbitrage return. Consequently, the balancing energy market will also attract additional capacity which is not tradable on the capacity reserve market. The declining amplitude of the quarter-hourly pattern is an indication of market participants recognizing and exploiting the balancing energy market in this manner. 
Figure 2: Expected quarter-hourly deviation conditional on the interval during a day (quarter-hourly pattern). Intervals forming an hour are joined by lines.

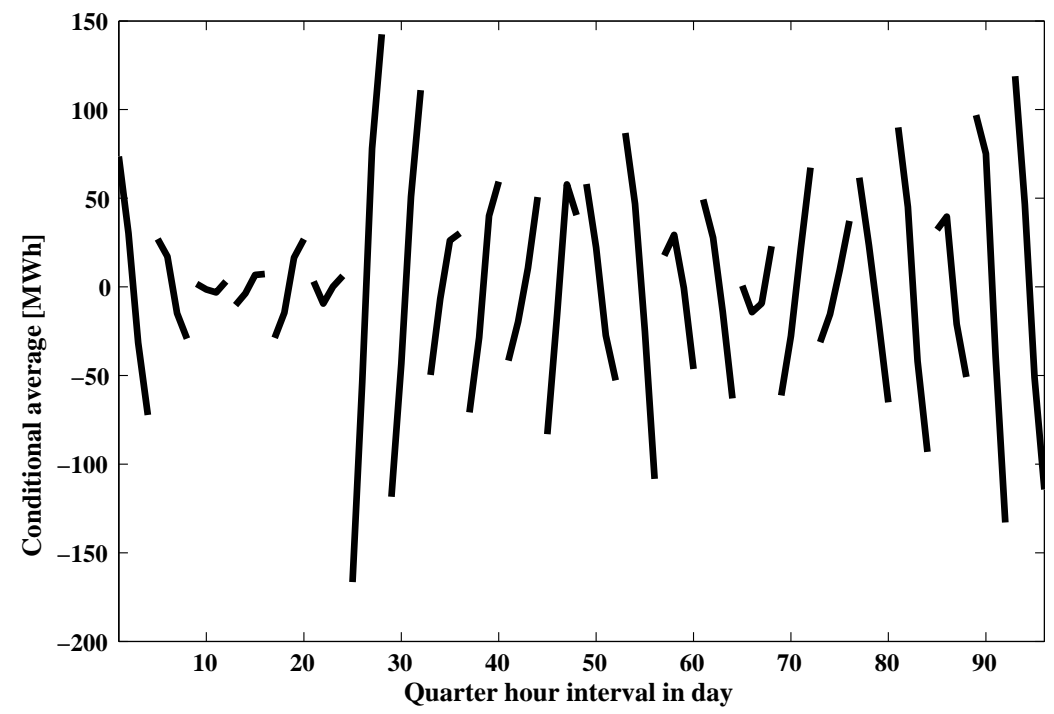

Figure 3: Comparison of model (light) and scaled data (dark) using 2004 data.

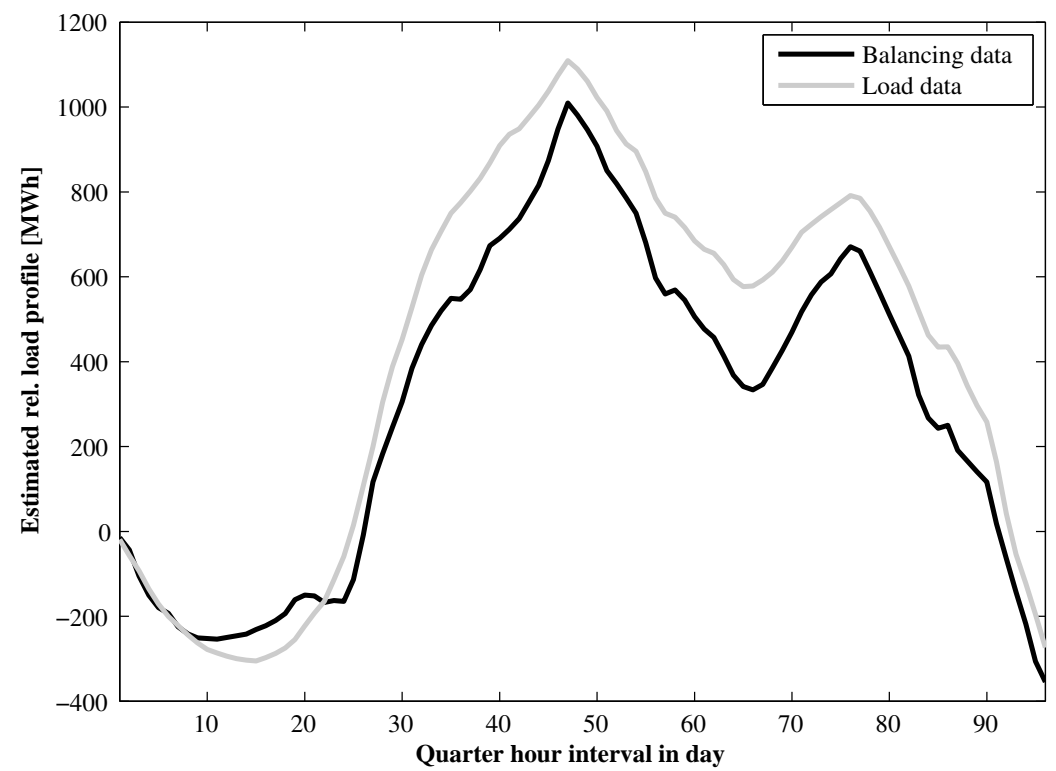


Table 1: $R^{2}$ using yearly quarter-hourly pattern and scaled total quarter-hourly pattern for prognosis

\begin{tabular}{|c|c|c|c|c|c|c|c|}
\hline \multirow{2}{*}{\multicolumn{2}{|c|}{$\begin{array}{c}\text { Predictor } \\
\text { pattern }\end{array}$}} & \multicolumn{6}{|c|}{ Year } \\
\hline & & 2003 & 2004 & 2005 & 2006 & 2007 & 2008 \\
\hline \multirow{7}{*}{$R^{2}$} & 2003 & - & 0.9853 & 0.9074 & 0.9413 & 0.8867 & 0.7830 \\
\hline & 2004 & 0.9865 & - & 0.9393 & 0.9520 & 0.9130 & 0.8427 \\
\hline & 2005 & 0.9396 & 0.9569 & 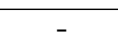 & 0.9738 & 0.9681 & 0.9329 \\
\hline & 2006 & 0.9538 & 0.9588 & 0.9683 & - & 0.9775 & 0.9260 \\
\hline & 2007 & 9243 & 0.9367 & 0.9673 & 0.9809 & - & 0.9703 \\
\hline & 2008 & 0.8746 & 0.9010 & 0.9405 & 0.9457 & 0.9743 & 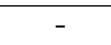 \\
\hline & Total $_{\text {scaled }}$ & 0.9820 & 0.9861 & 0.9896 & 0.9909 & 0.9866 & 0.9667 \\
\hline \multicolumn{2}{|c|}{ Scale $_{\text {in-sample }}$} & 1.1478 & 1.1025 & 0.9301 & 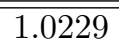 & 0.9405 & 0.8657 \\
\hline
\end{tabular}

Figure 4: Shape of the load curve as estimated from quarter-hourly balancing energy data

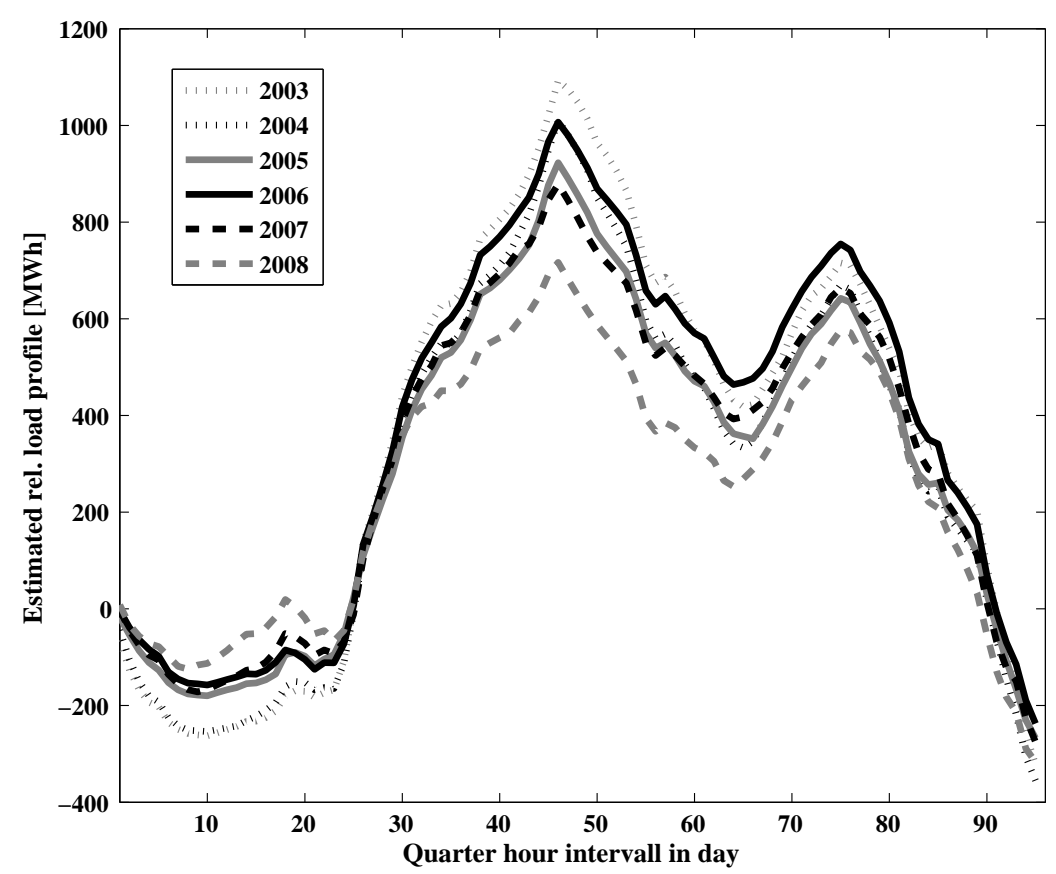




\section{Hourly pattern}

On an hourly timeframe the balancing energy is an alternative marketplace for the electricity trades in the day-ahead market and contracts in the capacity reserve market (see Section 2). To investigate the balancing energy data on this timeframe, we integrate the data to hourly values, which correspond to the hourly contracts traded in the day-ahead market. All subsequent analysis is based on these hourly balancing energy data.

As introduced in Section 4, there is a fundamental weekly seasonality in the German electricity market (see Figure 1). To match this seasonality we extract a weekly pattern from the balancing energy data using the following approach. For each day of the year a symmetric time window of seven weeks is applied, and the balancing energy data are aggregated over the years 20032008. The pattern is then estimated by the average demand, conditional on the hour within a week (see Figure 5). When compared to the load (see Figure 1), a similar seasonality is inherent in the balancing energy demand. The hourly balancing energy pattern is capturing both the weekly peak and off-peak shape, and the summer-winter dependence, well characterized by the presence of an additional pronounced demand peak during evening hours in the winter months. The presence of this pattern is clearly incompatible with all BRPs providing a balanced minimal variance forecast, as such forecasting should result in a purely random pattern. In general, the observed hourly positions result either from a reluctance of market participants to provide balanced forecasts, or they indicate intentional strategic behavior. An inadequate consideration of transmission losses and a load-dependent outage risk could be the reason behind the former. The latter is linked to arbitrage incentives between the balancing energy market and the day-ahead market, as outlined in Section 3. While it is impossible to disregard the reluctance of market participants, the following analysis demonstrates that the detected positions are at least partially of strategic nature.

To test the continuity of the hourly pattern, yearly patterns are calculated. In this the data are averaged over summer and winter months, so the resulting patterns will not uncover the summer-winter dependence. However, we compare individual years by using the out-of-sample average pattern for each year as a prediction for the in-sample pattern (see Table 2). With the exception of 2004, this simple model has reasonable predictive power. A further inspection of the prediction error in 2004 shows that the prediction captures the general shape well. However, it overestimates the amplitude. This finding is supported by an in-sample fit of a scale parameter to the out-of-sample pattern reported as $R^{2^{*}}$. The scale parameter of 2004 is almost halved. Reluctance does not explain such sudden changes in the observed pattern as it would change gradually if at all. So this reduced scale indicates a change in strategic positions in the balancing energy market. What is more, 2004 was a year with an exceptionally low number of electricity price spikes in the day-ahead market. In this context the absence of spikes reduces the arbitrage incentive between the balancing energy market and the day-ahead market, and may explain the change in amplitude of 
the hourly pattern.

Let's consider the discussion of balancing energy and day-ahead prices in Section 3 further. German balancing energy prices depend on the prices of secondary and tertiary reserve capacity and the balancing energy demand, while the price in the day-ahead market reflects the supply and demand equilibrium down to an hourly time scale. As secondary and tertiary reserve capacity prices involve longer contractual periods - one month and four hours - demand remains the sole determining factor of balancing energy prices responsive on an hourly timeframe.

In practice, market participants have an economic incentive to consume more of the risky, but evenly priced balancing energy, as demand and prices on the day-ahead market rise. Therefore, an hourly pattern in balancing energy should resemble the load profile. That is, market participants will exercise their grid excess as a real option when electricity prices are high, as long as balancing energy is expected to have a favorable price (i.e., until the two markets reach an equilibrium). We stress that exploiting this spread between day-ahead market prices and expected balancing energy prices is a statistical-arbitrage opportunity, as balancing energy prices are uncertain at the time a position is entered.

We introduce a factor $(I(t))$ to capture this incentive for strategic positions in the balancing energy market. This factor is defined by the difference of dayahead prices from the current price level. As a specification of the price level, we use the median price of the preceding four weeks. The time span of four weeks is chosen in an effort to balance stability and slackness considerations in the definition of a price level. This is supported by testing other multiples of weekly time spans that did not change the substance of the results. However, the median was explicitly chosen to create a spike-insensitive measure for the price level, so the defined factor will capture price spikes.

The marks in Figure 6 show the mean balancing energy demand conditional on our factor value. Here the balancing energy demand is measured relative to a long-term mean level of four weeks. This separates effects of longer time duration, which we will discuss in Section 7 . Each individual year in the dataset is displayed, demonstrating a continuous structure. The dependence structure reaches from a central linear domain into a domain of saturation at higher factor values. The effect of saturation is to be expected in view of the limited reserve capacity the grid operator provides (i.e., constraints imposed by network stability considerations and grid access contracts). In principle, these findings apply as well to all four control areas individually (see Figure 7). However, the data cannot account for balancing activity between control areas. Such effects are excluded by netting all four control areas, and we therefore restrict the further investigation to the hypothetical combined control area.

We propose a three-parameter factor model for the hourly balancing energy deviation pattern (see first summand in equation (3)):

$$
h\left(I(t), I_{\text {tec }}(t)\right)=a \cdot\left(\frac{2}{1+b \cdot e^{-c \cdot I(t)}}-1\right)+I_{\text {tec }}(t) \forall a \in \mathbb{R} b, c \in \mathbb{R}_{+}
$$


Figure 5: Weekly balancing energy pattern (Monday to Sunday) in summer (light) and winter (dark) (hourly pattern)
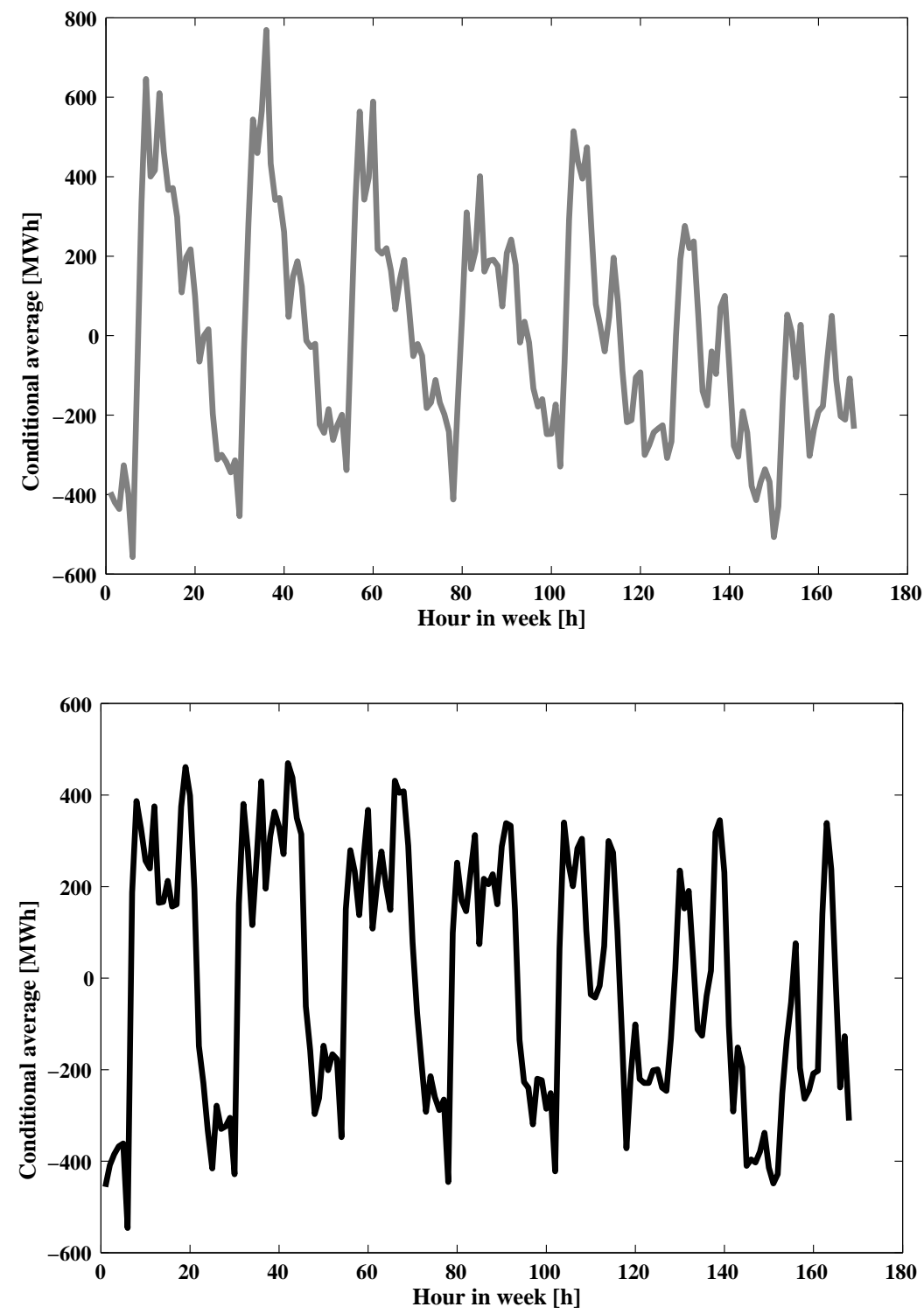
Table 2: $R^{2}$ using out-of-sample average and scaled out-of-sample average for prediction of the hourly pattern

\begin{tabular}{l||c|c|c|c|c|c} 
Year & 2003 & 2004 & 2005 & 2006 & 2007 & 2008 \\
\hline \hline$R^{2}$ & 0.7857 & 0.2003 & 0.7435 & 0.7712 & 0.8316 & 0.8492 \\
\hline$R^{2^{*}}$ & 0.7863 & 0.6229 & 0.8019 & 0.8286 & 0.8381 & 0.8536 \\
\hline \hline Scale $_{\text {in-sample }}$ & 0.9733 & 0.5483 & 0.7874 & 1.3570 & 1.0961 & 1.0772
\end{tabular}

Figure 6: Factor value $(I(t))$ and hourly balancing energy demand

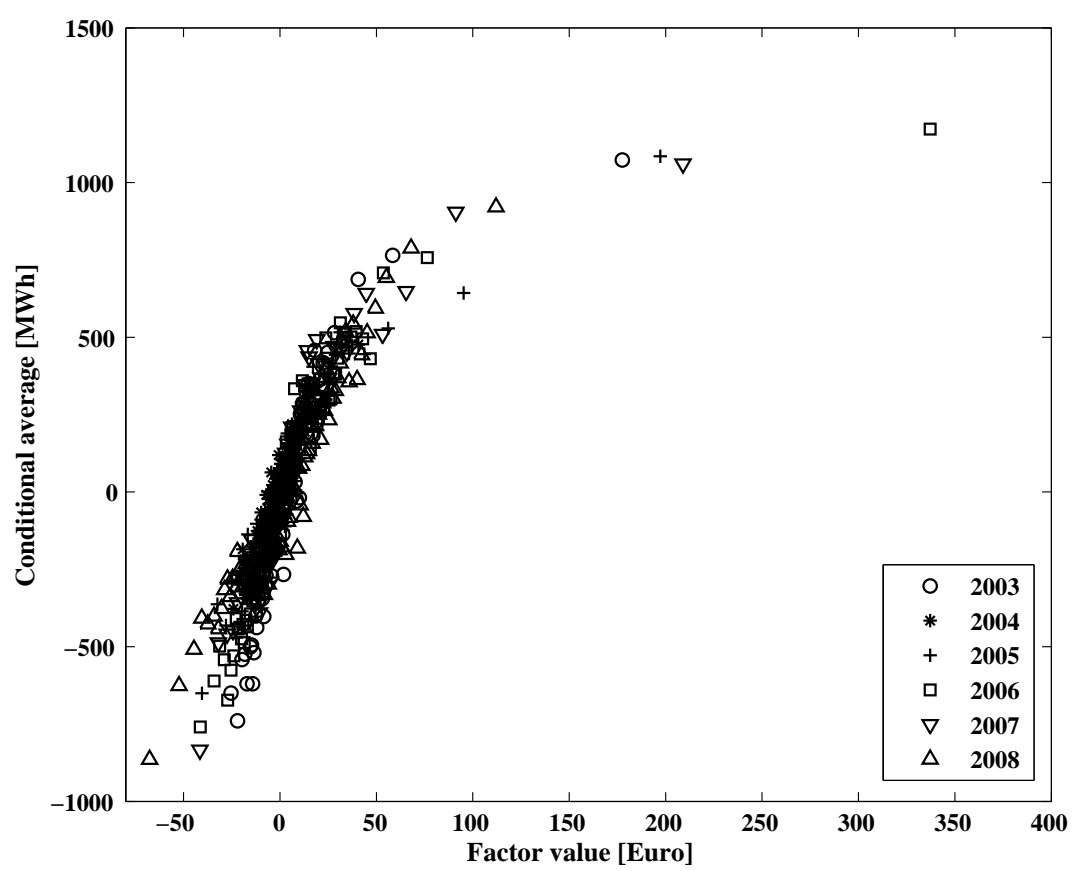


Figure 7: Conditional average hourly balancing energy demand versus factor value $(I(t))$ in the four German control areas
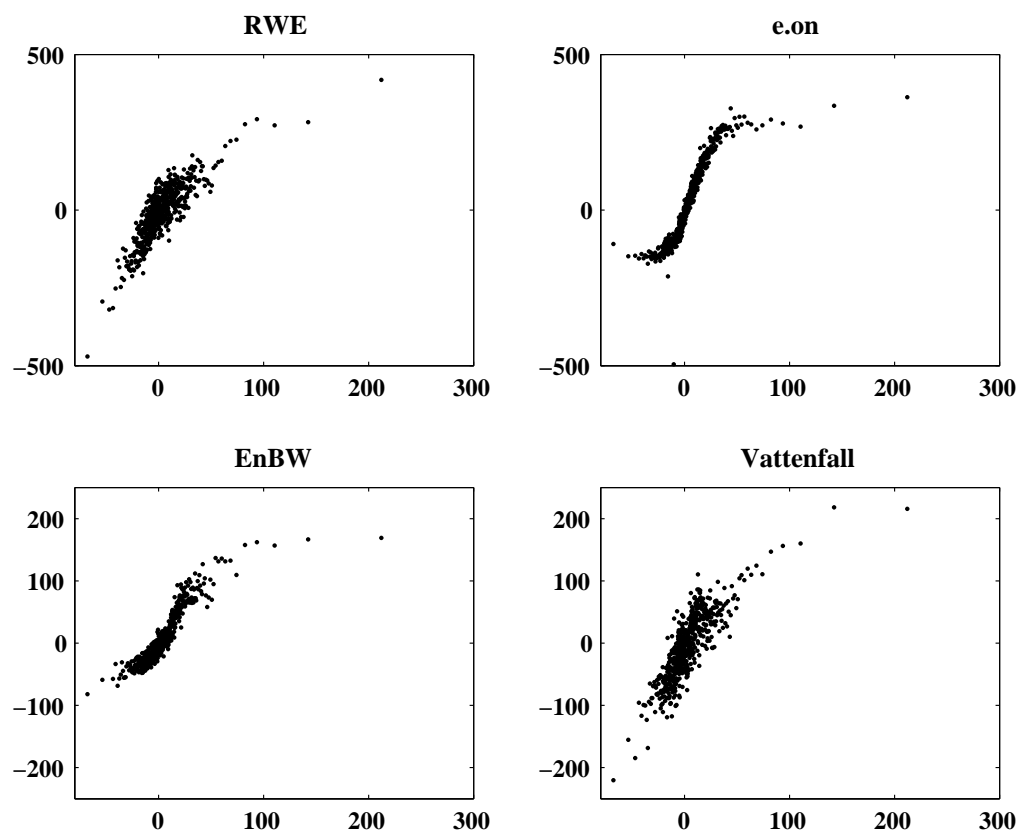
Figure 8 and Table 3 show parameter estimates from out-of-sample fits for each year and corresponding $R^{2}$ values. Evidently, the dependence structure is constant over time. Moreover, this factor model captures the change in amplitude, introduced by less volatile electricity prices in 2004 .

In view of the $R^{2}$ values in Table 2, the factor model does not seem to explain the hourly pattern fully. A further inspection of the residual pattern shows the change in amplitude for the year 2004 to be captured well, but some pronounced seasonal effects remain. One such example is a highly negative balancing energy demand between five and six at weekday mornings. This coincides with peak units going online to cover the following steep ramping hours. To include such very technical effects that will be constant over time, we use the out-of-sample weekly average pattern as an additional factor $\left(I_{\text {tec }}(t)\right)$. The resulting combined model in equation (3) can explain much of the detected seasonal variation (see Table 3). Also when compared to the $R^{2^{*}}$ values in Table 2, the combination of arbitrage incentive and technical incentive shows similar predictive power. However, the latter model does not resort to in-sample information. Using this out-of-sample prediction, the variance of the hourly balancing energy data is reduced by $19.2 \%$.

The detected hourly pattern can be modeled by equation (3). While the $I_{t e c}$ component in this model is compatible with a reluctance of market participants to provide a balanced forecast, the contribution of the arbitrage incentive is evidence of strategic balancing energy deployment. Clearly market participants recognize and implement the arbitrage opportunities between the day-ahead market and the balancing energy market in their portfolio management. Such strategies result in a lower than average amplitude of the hourly balancing energy pattern in years with less than average electricity price spikes as 2004 . 
Figure 8: Factor model $(I(t))$ prediction and data

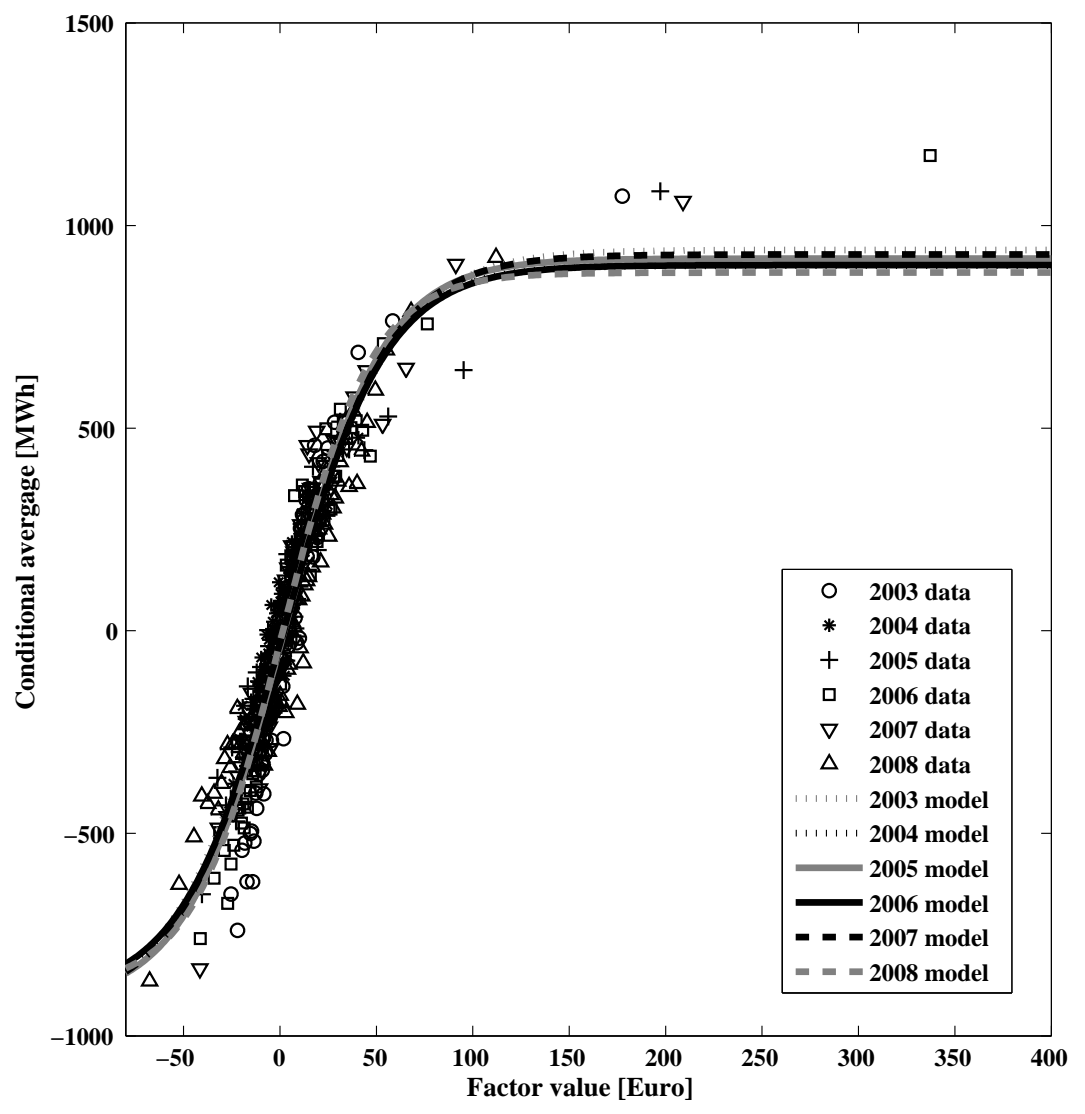

Table 3: Hourly pattern: parameters and $R^{2}$ fitting to out-of-sample data

\begin{tabular}{c||c|c|c||c|c}
\multicolumn{1}{l||}{} & \multicolumn{3}{c||}{ Parameters } & \multicolumn{2}{c}{$R^{2}$ factor model } \\
Year & $a[M W h]$ & $b[E]$ & $c[1 / €]$ & $I(t)$ only & $I(t)$ and $I_{t e c}(t)$ \\
\hline \hline 2003 & 940.045 & 1.053 & 0.035 & 0.6948 & 0.7252 \\
\hline 2004 & 901.082 & 1.113 & 0.039 & 0.4448 & 0.6170 \\
\hline 2005 & 918.633 & 1.089 & 0.039 & 0.6069 & 0.7518 \\
\hline 2006 & 902.485 & 1.072 & 0.038 & 0.8424 & 0.8499 \\
\hline 2007 & 928.798 & 1.081 & 0.037 & 0.7571 & 0.7998 \\
\hline 2008 & 884.110 & 1.068 & 0.043 & 0.6530 & 0.7025
\end{tabular}




\section{Long-term pattern}

After a few of their respective cycles, the average of both the quarter-hourly pattern and the hourly pattern is zero. In order to complete the analysis, we look at positions in the balancing energy market that are persistent over longer periods of time. We extract these positions from the residuals of the hourly factor model (see equation (3)), by application of a seasonal autoregressive integrated moving average (SARIMA) model. For this model we resort to the results of Möller et al. (2009), which we briefly present.

Möller et al. (2009) suggest the SARIMA- $(1,0,0) \times(1,0,1)_{24}$ model given by equation (4).

$$
y_{t}=a_{1} y_{t-1}+a_{24} y_{t-24}-a_{1} a_{24} y_{t-25}+b_{24} \sigma \epsilon_{t-24}+\sigma \epsilon_{t}, \quad \epsilon_{t} \in t(\nu)
$$

In a comparison of the parameter estimates using data of the individual years, similar parameter sets are found over the entire time span. We therefore use the total sample estimates in the remainder of this analysis. Additionally, the innovation process shows heavy-tailed effects, which are modeled by a classical tempered stable (CTS) distribution see, for example (Kim et al., 2008; Menn and Rachev, 2009).

Finally, the forecasts of the model are tested on two relevant time horizons, which reflect the information disclosure on the balancing energy market in Germany. It is found that the variance is reduced by applying a three-day lag in information disclosure as well as using a one-month time horizon in forecasting. Moreover, the yearly average value of these forecasts deviates from zero (see Figure 9).

Let's consider these results in the context of electricity portfolios. Market participants use the balancing energy market not only for short-term adjustments to their portfolio, but also to take positions over extended periods of time. This view is supported by Figure 9, which shows the timely evolution of the three-day forecast. It clearly displays the predictable long-term offset in balancing energy demand. Also the magnitude and the sign of this offset vary over the years. It persists even in the case of the long forecast horizon of a month. So this offset cannot be attributed to a lack of information, and the change in amplitude and sign indicates intentional positions.

Like in the case of the hourly pattern, a long-term position in the balancing energy market coincides with a countering position in the futures market. Specifically we use the day-ahead futures market as a reference. From this perspective, the price of the deviation is the difference between balancing energy price and day-ahead market price. Or, in other words, a positive deviation can be described as a short position in a day-ahead contract, and a long position in the balancing energy market, and vice versa for a negative deviation. In turn, the cost of deviation is obtained by multiplying price and volume, and the cost function can be approximated by the average cost for a given deviation.

Figure 10 shows the estimated cost function in the different control areas using 2003-2008 as the sample data. At a deviation close to zero, the cost increases linearly, indicating a constant price. However, the cost function levels for large 
Figure 9: Prediction values with a three-day lag in information disclosure

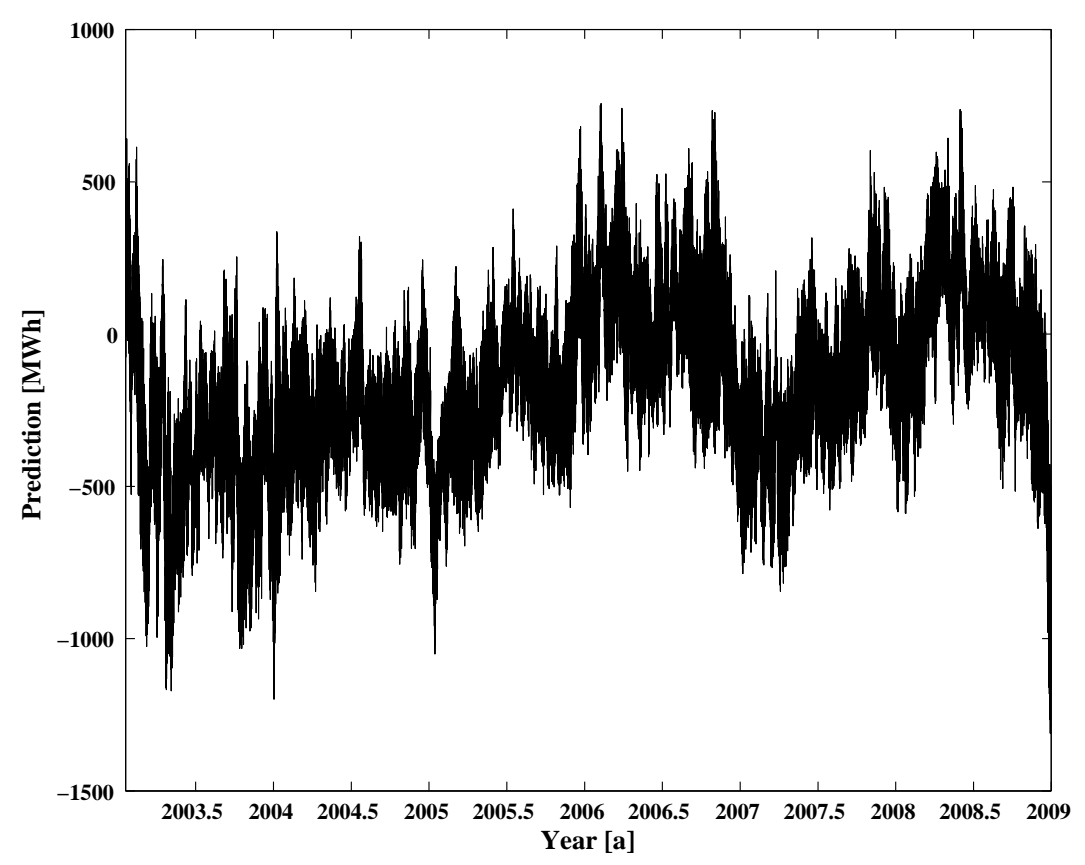


negative deviations, whereas it increases drastically at large positive deviations. This asymmetry in the cost function is an important point. Consider a strategic position in the balancing energy market. It displaces the location parameter of the forecast error, while scale and higher moments will not be affected. Under the described cost function, shifting the deviation towards the negative (i.e., a surplus of day-ahead contracts) will continuously incur cost from additional negative deviation, while reducing the risk of "unbounded" cost at high positive deviation. So given an unavoidable forecast error, a negative net position is a rational response to the observed cost function.

For a further inspection, we concentrate on the largest control area, in terms of load, the RWE control area. Looking closer at the cost function of individual years, the slopes of the cost function vary (see Figure 11). Particularly interesting is the difference in slope for positive and negative deviations within individual years. A difference in slope provides an incentive to move deviation risk towards the flatter side of the cost function in order to reduce cost. In the example of the RWE control area, the cost functions for the years 2005 and 2006 indicate an incentive towards positive deviation, while for the other four years investigated a negative net deviation would have been profitable. Finally, the opening angle between the linear domains at the positive and the negative branch of the cost function varies. Here, a wider opening angle will incur less cost for a strategic deviation.

Using these arguments, the increasing long-term position in 2005, 2006, and 2008 (see Figure 9) is an adequate adaption to a cost function tilting towards positive deviation. In the cases of 2003, 2004, and 2007, a negative position coincides with a cost function tilted towards negative deviation. Additionally, the opening angle of the cost function narrowed in 2008, providing an incentive to reduce strategic positions.

A complete investigation should include all four control areas. However, these findings demonstrate that there are economic incentives behind the detected long-term balancing energy positions. With its asymmetric cost function for the balancing energy, the German market design appears being prone to push market participants to a strategic short position in the balancing energy market. These positions have to be countered by a long position in the futures markets. In other words, the German market design creates a virtual demand in the day-ahead market. 
Figure 10: Expected cost of deviation [€] versus deviation [MWh] in the four German control areas
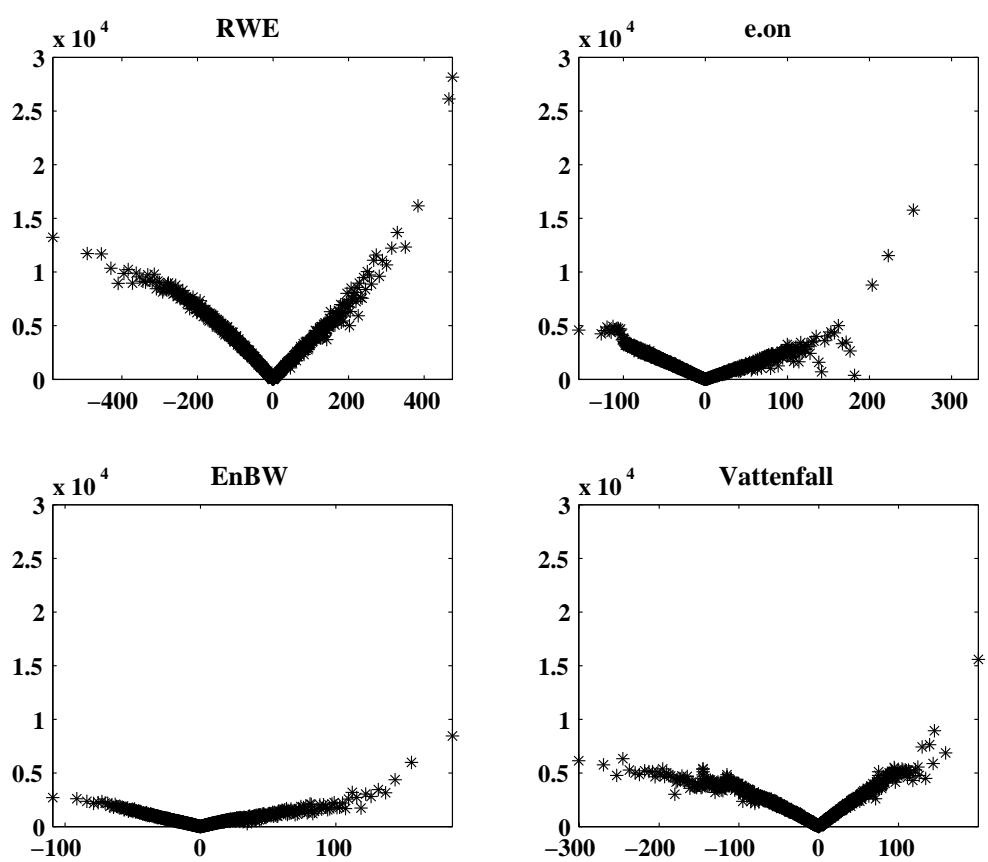
Figure 11: Empirical cost function of deviation $[€]$ versus deviation [MWh] in RWE control area from 2003 to 2008
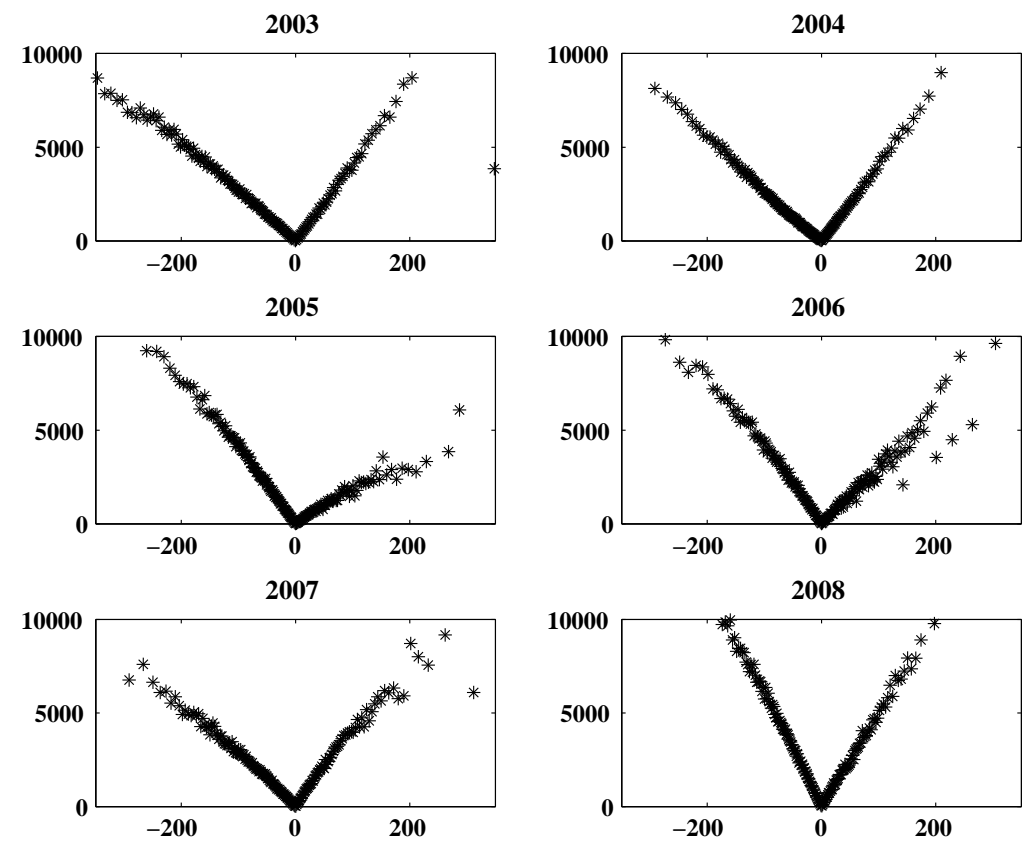


\section{Conclusion}

In this paper we provide a model for balancing energy demand in the German electricity market. This model is built on the notion of the balancing energy market as an alternative marketplace to both the day-ahead market and the capacity reserve market. Corresponding to these marketplaces we identify three strategies used on different time scales.

Within the hour, different settlement periods in the day-ahead and balancing energy market lead to a pronounced quarter-hourly pattern. A high spread between up- and down-regulation balancing energy prices in different periods translates into an economical incentive to obtain less correlation to this quarter-hourly pattern. This strategy reduces load fluctuation in the network, and therefore provides an alternative to market capacity reserve. In this way the balancing energy market design attracts reserve capacity, in addition to the capacity traded on the reserve capacity market, where pre-qualification standards apply and constitute a restriction to market access.

On an hourly timeframe, a pattern resembling the load curve is identified. This hourly pattern shows that market participants exploit statistical arbitrage opportunities between the balancing energy market and the day-ahead market. In other words, the hourly pattern can be understood as the exercise of gridaccess as a real option. The presence of this strategy shows that the market responds to the incentives set by the market design, and indicates balancing energy to be an integral component of electricity portfolio management in Germany.

Additionally, we identify positions taken in the balancing energy market over extended periods of time. Changes in these positions coincide with changes in the asymmetric cost function of balancing energy. This observed asymmetry provides an economic incentive to provide an unbalanced forecast and reduce deviation cost. Historically, the asymmetry displays a tendency to drive the market towards oversupply. In this context the authorization of negative prices in Germany in 2008 constitutes a potential turning point. Future research will show if the observed tendency to an over supplied market prevails.

In theory, the observed long-term and hourly balancing energy positions might adversely affect network stability. However, from a historical perspective, there is no indication of these positions jeopardizing network stability see (Nailis and Ritzau, 2006). At the same time, the German balancing energy market design offers interesting properties, especially with a view on an extension of weather-dependent renewable generation and its associated challenges. These challenges include the supply of adequate reserve capacity to manage weather-induced fluctuations. The German market design complements this need - if not directly, then indirectly by releasing other capacity - in providing a marketplace for reserve capacity otherwise shielded from the market. Another challenge is the phenomenon of electricity price spikes, resulting from weatherinduced supply shortages. The effects of such spikes on electricity portfolios are mitigated by the balancing energy market as an alternative marketplace to the day-ahead market. 
In this light, the balancing energy market contributes to an efficient use of resources, and enables markets to incorporate a higher share of renewable generation. The model is an important element in implementing the described strategies and managing associated risk. If fully exploited, the model reduces balancing energy demand by $12 \%, 19 \%$, and $11 \%$ on the three respective timeframes. The released reserve capacity can then be allocated to account for a further extension of renewables. 


\section{References}

Geman H, Roncoroni A. Understanding the fine structure of electricity prices. Journal of Business 2006; 79 (3); 1225-1261.

Trück S, Bierbrauer M, Menn C, Rachev S. Spot and derivative pricing in the EEX power market. Journal of Banking and Finance 2007; 31; 3462-3485.

Römisch, Wegner-Specht. Modellierung der Spotpreise - Literaturüberblick. Literaure review; Humboldt-Universität zu Berlin; part of SIMOPT/ENERGY project; 2005.

Bessembinder H, Lemmon M. Equilibrium pricing and optimal hedging in electricity forward markets. Journal of Finance 2002; 57 (3); 1347-1382.

de Jong C, Huisman R. Option formulas for mean-reverting power prices with spikes. Tech. Rep.; Energy Global and Erasmus University; 2002.

Weigt H, Riedel S. German electricity reserve markets. Electricity Markets Working Paper, SSRN 2007; Available at SSRN: http://ssrn.com/abstract $=1137282$.

Simoglou C, Bakirtzis A. Electricity producer self-scheduling in day-ahead energy and reserves markets. In: European Electricity Market, 2008. EEM 2008. 5th International Conference; 2008.

ETSO, Balance management harmonisation and integration 4th report. Tech. Rep.; ETSO; 2007.

Belmans R, Deuse J, Meeus L, Purchala K, Stubbe M, Vandezande L. Study on interaction and dependencies of balancing markets, intraday trade and automatically activated reserves. Tech. Rep.; Tractebel Engineering and Katholieke Universiteit Leuven (KUL); 2009.

ERGEG. Guidelines of good practice for electricity balancing markets integration. Tech. Rep.; European Regulators Group for Electricity and Gas; ref: E05-ESO-06-08; 2006.

EU. 2020 by 2020 Europe's climate change opportunity. Tech. Rep.; Commission of the European Communities; $\operatorname{COM}(2008) 30$ final; 2008.

U.S.Congress. American clean energy and security act of 2009. Tech. Rep.; 111th United States Congress; passed the House on June 26, 2009; 2009.

EU. Technical annexes to the report from the commission on the implementation of the gas and electricity internal market $\operatorname{COM}(2004) 863$ final. Commission Staff Working Document; Commission of the European Communities; 2005.

Kirschen D, Garcia M. Market clearing prices in the balancing mechanism of England and Wales. In: Power Systems Conference and Exposition, 2004; 2004 . 
Mielczarski W, Siewierski T, Wedzik A. Experience from the operation of the Polish electricity market. Power Tech, 2005 IEEE Russia 2005; 1-7.

Bundesnetzagentur. Bilanzkreisvertrag über die Führung eines Bilanzkreises. http://www.bundesnetzagentur.de; 2006.

RWE Transportnetz Strom. Bilanzkreisabweichungen. https://www.regelleistung.net; 2009.

e.on Netz, Bilanzkreisabweichung, https://www.regelleistung.net, 2009.

EnBW Transportnetze. Bilanzkreisabrechnung: Verfahren und Preise. https://www.regelleistung.net; 2009.

Vattenfall Europe Transmission. Ausgleichsenergie Arbeitspreise. https://www.regelleistung.net; 2009.

University of Karlsuhe IIP. Quarter hourly load data of Germany in 2004. 2008.

Nailis D, Ritzau M. Studie zur Marktausgestalltung der Regel- Ausgleichsenergie vor dem Hintergrund des neuen EnWG. Tech. Rep.; BET; 2006.

Möller C, Rachev S, Kim Y, Fabozzi F. Innovation processes in logically constrained time series. Tech. Rep.; Universität Karlsruhe (TH)- ETS; 2009.

Kim Y, Rachev S, Bianchi M-L, Fabozzi F. Financial market models with Levy processes and time-varying volatility. Journal of Banking and Finance 2008; 1363-1378.

Menn C, Rachev S. Smoothly truncated stable distributions, GARCH-Models, and option pricing. Mathematical Methods in Operation Research 2009; 69 (3); 411-438. 